\title{
Challenges in the Packaging of MEMS
}

(1*) Ajay P. Malshe

High Density Electronics Center (HiDEC)

Department of Mechanical Engineering

204 Mechanical Engineering Building

University of Arkansas

Fayetteville, AR 72701-1201

Phone: (501) 575-6561

Fax: (501) 575-6982

e-mail: apm2@engr.uark.edu

$\left(3^{*}\right)$ Sushila B. Singh

High Density Electronics Center (HiDEC)

Department of Mechanical Engineering

204 Mechanical Engineering Building

University of Arkansas

Fayetteville, AR 72701-1201

Phone: (501) 575-7250

Fax: (501) 575-6982

e-mail: ss22@engr.uark.edu

$\left(5^{*}\right)$ William P. Eaton

Reliability Physics Department

Sandia National Laboratories .

PO Box 5800

Albuquerque, NM 87185-1081

Phone: (505) 845-8209

Fax: (505) 844-2991

e-mail: weaton@sandia.gov $\left(2^{*}\right)$ Chad O'Neal

High Density Electronics Center (HiDEC)

Department of Mechanical Engineering

204 Mechanical Engineering Building

University of Arkansas

Fayetteville, AR 72701-1201

Phone: (501) 575-4609

Fax: (501) 575-6982

e-mail: nco@engr.uark.edu

$\left(4^{*}\right)$ William D. Brown

High Density Electronics Center (HiDEC)

Department of Electrical Engineering

3217 Bell Engineering Center

University of Arkansas

Fayetteville, AR 72701-1201

Phone: (501) 575-6045

Fax: (501) 575-7967

e-mail: wdb@engr.uark.edu

$\left(6^{*}\right)$ William M. Miller

Reliability Physics Department

Sandia National Laboratories

PO Box 5800

Albuquerque, NM 87185-1081

Phone: (505) 844-7161

Fax: (505) 844-2991

email: millerwm@sandia.gov

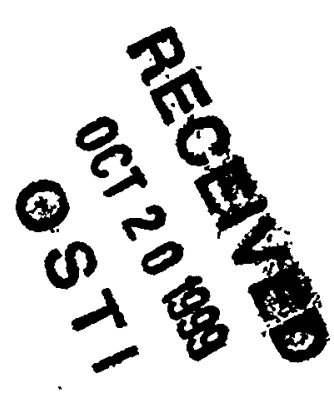

* Order in which the authors should be included in the final paper.

\section{Abstract}

Microelectromechanical Systems (MEMS) packaging is much different from conventional integrated circuit (IC) packaging. Many MEMS devices must interface to the environment in order to perform their intended function, and the package must be able to facilitate access with the environment while protecting the device. The package must also not interfere with or impede the operation of the MEMS device. The die attachment material should be low stress, and low outgassing, while also minimizing stress relaxation over time which can lead to scale factor shifts in sensor devices. The fabrication processes used in creating the devices must be compatible with each other, and not result in damage to the devices. Many devices are application specific. requiring custom packages that are not commercially available. Devices may also need media compatible packages that can protect the devices from harsh environments in which the MEMS device may operate. Techniques are being developed to handle, process, and package the devices such that high yields of functional packaged parts will result. Currently, many of the processing steps are potentially harmful to MEMS devices and negatively affect yield. It is the objective of this paper to review and discuss packaging challenges that exist for MEMS systems and to expose these issues to new audiences from the integrated circuit packaging community.

\section{Introduction}

Microelectromechanical Systems (MEMS) consist of mechanical devices and machine components ranging 


\section{DISCLAIMER}

This report was prepared as an account of work sponsored by an agency of the United States Government. Neither the United States Government nor any agency thereof, nor any of their employees, make any warranty, express or implied, or assumes any legal liability or responsibility for the accuracy, completeness, or usefuiness of any information, apparatus, product, or process disclosed, or represents that its use would not infringe privately owned rights. Reference herein to any specific commercial product, process, or service by trade name, trademark, manufacturer, or otherwise does not necessarily constitute or imply its endorsement, recommendation, or favoring by the United States Government or any agency thereof. The views and opinions of authors expressed herein do not necessarily state or reflect those of the United States Government or any agency thereof. 


\section{DISCLAIMER}

Portions of this document may be illegible in electronic image products. Images are produced from the best available original document. 
in size from a few microns to a few hundred microns. They can be the mechanical interconnects of microsystems, and can also transduce signals from one physical domain to another, such as mechanical-to-electrical, electrical-tomechanical, electrical-to-chemical, etc. These devices are broadly categorized as either sensors or actuators.

MEMS sensors are devices such as pressure sensors, accelerometers, and gyrometers that perceive an aspect of their environment and produce a corresponding output signal.

Actuators are devices that are given a specific input signal on which to act and a specific motion or action is produced. For example, Figure 1 shows a scanning electron micrograph of a microengine connected to a geartrain. Other examples of MEMS actuators are microengines, microlocks, and discriminators ${ }^{1.2}$. Sensors can be thought of as being passive, waiting on a signal from the environment to elicit a response, while actuators are activated by the user. The sources of motion for MEMS elements such as gears or microlocks are usually electrostatics, thermoactuation, wobble motors, and with limited success, even microsteam engines'. These "motors" provide the mechanical input required to activate the actuators and can be used with a gear train to raise or lower a micromirror for a digital light processing application ${ }^{2}$. These microengines produce the motion of a linear actuator or activate a weapon safety system that requires motion of the mechanical locking elements ${ }^{3-5}$. Just these few examples show the diversity of interfaces that MEMS packaging engineers face between signal domains.

In state-of-the-art manufacturing, MEMS are fabricated using manufacturing processes and tools borrowed from the microelectronics industry. Many of these processes and tools are used directly, while some have been modified to meet the specific needs of MEMS ${ }^{6}$. The development cycle for MEMS is long and often several design cycles are required to comply with design specifications. This is because, in many cases, the products, as well as the technologies are being developed simultaneously. MEMS design and fabrication are more like that of application specific integrated circuits (ASICs). There are currently relatively few generic parts that can be rearranged to create any one of thousands of different devices in the manner that resistors, transistors, and capacitors can be arranged in integrated circuit (IC) design ${ }^{7}$. This can be problematic for developing MEMS packaging since every device is different in fabrication and application. Research laboratories like Sandia National Laboratories and Cronos Integrated Microsystems (a subsidiary of $\mathrm{MCNC}$ ) are developing libraries of different parts that can be reassembled in various configurations ${ }^{8.9}$. Researchers are also working on CAD (computer aided design) tools for MEMS that would facilitate solid modeling of these MEMS devices, reducing design cycle times. This allows faster design times, and increased modularity of the devices.

As in MEMS fabrication, MEMS packaging borrows many processes and toolsets. However, extra care must be taken since MEMS dice need to interface with the environment for sensing, interconnection, and/or actuation $^{10}$. MEMS packaging is application specific and the package allows the physical interface of the MEMS device to the environment ${ }^{10}$. In the case of a fluid mass flow control sensor, the medium flows into and out of the package. Harsh environments may create different challenges for the packaging of MEMS ${ }^{10}$. These types of packaging are referred to as media compatible packaging. In addition to challenges related to the environment of the MEMS chip and interfacing it with the environment, challenges also exist inside the MEMS package with die handling, die attach, interfacial stress, and outgassing". These new challenges in the field of MEMS packaging need immediate research and development efforts. The reason for this attention is that devices can easily be destroyed by assembly and packaging processes. If a device no longer performs its intended function, whether it be rotary actuation or pressure sensing, it is simply defined as failure.

To date, most of what is known about MEMS packaging remains proprietary and published literature is scarce. The challenges of MEMS packaging have been known for some time, but little open research has been done to collect data and work toward meeting these challenges". A disproportionality exists between the resources spent on the packaging of MEMS and the time spent researching MEMS packaging. Currently, the cost of MEMS packaging typically accounts for $75 \%$ or more of the sale price of the device ${ }^{12}$. In contrast with IC packages, which are considered commodity items, many MEMS packages will be specialty items that will generate higher profit margins. This paradigm-shift will create new opportunities for small, low-volume packaging companies.

MEMS packaging is extremely important for the viability of MEMS systems' success. New devices are continually being developed that have great promise for miniaturizing existing sensors and actuators, as well as novel devices not previously possible. Currently it is the packaging of these devices that is limiting their market applications. MEMS devices that are currently available such as pressure sensors and accelerometers are devices that have been relatively simple to package, and therefore, have commercial viability. MEMS is a few billion dollar market today, and is looming on the edge of revolutionizing the entire world with products that are both smaller and less expensive than current products in the sensor and actuator regime. MEMS has been called the second silicon revolution, with benefits far beyond what has been seen with the first silicon revolution in integrated circuit 
technologies. Fabrication techniques, equipment and new technologies are evolving every year that advance the field of MEMS even further. It is of the highest importance that MEMS packaging stay abreast of the fabrication so that new systems can be both created and integrated into widespread commercial and military applications.

\section{Challenges in MEMS System integration}

There is a philosophical difference between the motivation for packaging ICs and packaging MEMS. The goal of IC packaging is to provide physical support for the chip, provide an electrical interface to active chip(s) in the system, supply signal, power, and ground interconnections, and allow heat dissipation ${ }^{13}$. Also, a package must effectively isolate the chip physically from its environment. MEMS devices, on the other hand, often are intimately interfaced with their environment ${ }^{10}$. Figure 2 shows a comparison between a typical DIP IC package and a MEMS pressure sensor package, which has an opening to sense pressure variation. Another issue is the media compatibility of the MEMS package. MEMS devices may need to operate in diverse environments or media such as under automobile hoods, with intense vibrations, and in salt water, strong acids, alkaline or organic solutions. For some pressure sensors, Media compatibility has been achieved by protective parylene coatings and silicone gel fills ${ }^{10}$. Overall, the package, while performing detection or actuation, must be able to withstand the environment(s).

Another challenge in MEMS packaging is the effect of packaging parameters on reliability. The package is part of the complete system and all aspects of the system must function together and must be compatible with each other. This determines which materials and what design considerations and limitations become important. One of the main scientific challenges of MEMS is the issue of material properties. The properties of the materials depend on how they are used, processed, the heat treatments to which the materials are subjected, and even the specific pieces of equipment used during fabrication. For instance, using a die attach material with a large coefficient of thermal expansion (CTE) mismatch with silicon can induce undesirable stress levels on the device. Not all the materials used react in the same way to these parameters, so compromises must be made. Some materials may be hard to obtain with research and development production run numbers. Low quantities of materials are used, and suppliers are reluctant to sell small quantities or develop new products for limited markets, such as protective media compatibility coatings". One favorable aspect about the materials used in microsystems is that the material properties generally get better at the microscale. This is due to a decrease in the number of defects encountered in the materials. The defect density remains about the same as in the macroscale, but since the MEMS devices are so small, the chance of a killer defect occurring in a device is reduced.

Packaging of MEMS dice is application specific, and hence, desired process steps could vary significantly. For example, a pressure sensor package will be dramatically different than a package for an micromirror array. Thus, it is important to classify MEMS dice by their packaging requirements and then develop the packaging standards and related knowledge base. Different standards would apply for different types of MEMS applications, since they operate in such a variety of different physical domains. For example, microfluidic devices have requirements that are vastly different than electrostatic actuators. The device and the package should be designed concurrently with the application and the environment in mind from the project conception.

Table I summarizes the different techniques that can be used to meet the various packaging parameter challenges. These solutions are not complete or foolproof, but are techniques that have been exercised and provide direction for further research. In the first column of the table are the packaging domains where challenges exist. The second column is the list of challenges that must be overcome for efficient batch processing of MEMS with high yield margins. The final column is a list of possible solutions that are being used, or could be used to meet the challenges in the center column. The following section goes into much more detail in discussing these challenges and possible solutions.

\section{Release and Stiction}

The process of freeing the die by etching oxide, so it becomes operational is referred as release of die. Releasing of the MEMS dice is an important step in MEMS packaging. Typically, the polysilicon fearures are surrounded by silicon dioxide during fabrication which protects the features and prevents them from becoming damaged or contaminated. Before using, this oxide is etched away, freeing the devices so they become operational. This is done using an $\mathrm{HF}$ etch which is selective between $\mathrm{SiO}_{2}$ and $\mathrm{Si}^{3}$. The challenge exists in economical and efficient releasing of the MEMS parts. It is most economically done in wafer form as a batch process, but this leads 
to near-certainty that contamination of the devices and destruction of the parts during the dicing of the released wafer. The cooling fluid used in dicing will obliterate the tiny mechanical devices. The most inefficient time is after dicing since each chip is released individually rather than the entire wafer at once. The MEMS features, however, do remain protected throughout the potentially lethal dicing stage, and currently, this is the only way to obtain functional devices at the die level.

During and after the release there is possibility of pulling down the beam-type element to the substrate due to capillary forces which is termed as stiction. Stiction occurs from the capillary action of the evaporating rinse solution in the crevices between elements, like between cantilevers and the substrate ${ }^{14}$. As the device dries, surface tension pulls its features into contact with the substrate. Stiction can render the MEMS devices useless after all other resources have been invested in them. An example of stiction is shown is Figure 3. The beam-type element has been pulled down to the substrate by the capillary forces. Preventing stiction from occurring after release is a major challenge. Some methods that are effective are freeze drying and supercritical $\mathrm{CO}_{2}$ drying ${ }^{15}$. These methods remove the liquid surface tension from the drying process, preventing stiction from occurring. Although, not yet foolproof, these methods have been used with some success.

MEMS parts can also be placed in an additional bath that applies a monolayer film that reduces stiction on the surface of the devices. There are coatings of different compositions that can be used and have been proven successful in preventing stiction. There are issues, however, on the stability and integrity of these coatings. Little is understood about how these films age, and if they will still be present on the devices after a year or several years. Also, these films can be scrubbed-off by the friction between moving parts, where stiction is a major issue. Additional research is needed on the subject of these coatings that includes stability studies over time. Another method that can be implemented as a processing step is to roughen the surface of the devices to minimize contact area between features ${ }^{16}$. A major problem here is that the very areas susceptible to stiction are very difficult to process in this manner. The close tolerances and gaps between the features and the substrate make it impossible for dry etching methods to be effective. Stiction can also be reduced in the design process by using dimples in regions of the device where stiction may be a problem ${ }^{17.18}$. These small protrusions on the bottom of an element can greatly reduce the contact area between the MEMS device element and the substrate. These are simple cuts in the sacrificial oxide layers that allow polysilicon to form a bubble on the bottom side of the device feature. Figure 4 shows a cantilever beam with dimples on the bottom surface.

\section{Dicing}

Another challenge in MEMS packaging is dicing the wafer into individual dice. Dicing is typically done with a diamond saw a few mils thick. This requires that coolant flow over the surface of the very sensitive dice, along with silicon and diamond particles that are generated during sawing, and which are all deadly to the devices. These particles, combined with the coolant, can contaminate and even destroy the devices, ruining yield. The fluid simply washes the features off the surface of the wafer. Contaminants can also get into the crevices of the remaining features, causing those devices to fail. An alternative to dicing is wafer cleaving. Wafer cleaving, also known as the scribe and break technique, is common in III-V semiconductor lasers and also has applications in MEMS $^{19}$. Cleaving does not require coolant and does not generate nearly as many particles as sawing. However, cleaving is difficult to master, and is highly dependent on the crystal structure and direction of the wafer. Laser dicing and wafer-level encapsulation are two other methods that may decrease the hazards of dicing, but increase the cost of the processing and assembly. Laser dicing is very accurate and generates few particles, but may be expensive and slower than diamond sawing. Wafer-level encapsulation also increases cost and processing steps and will be discussed in more detail in a later section.

\section{Die Handling}

Die handling is another area of MEMS system fabrication that requires additional development. Because of the delicate surface features of MEMS, these dice cannot be handled from the top die surface using vacuum pickup heads as in traditional IC die assembly. The vacuum heads may damage the surface features of MEMS devices. The MEMS dice must be handled by their edges, which will require new infrastructure for the automated handling of MEMS. Handling chips by the edges is more difficult than by the top surface because of a greatly reduced surface area in the pick-up region and increased dexterity requirements of the pick-and-place equipment. Effective 
edge handling equipment could simply be collets that fit into existing pick and place equipment and allow the dice to be picked up by the edges using vacuum or mechanical clamping. These MEMS die handling fixtures could be fingers or clamps that delicately handle the MEMS dice by their edges. In order to handle the high volumes of MEMS chips, die handling fixtures and methods that handle the chips by the edges must become commonplace in intermediate to high volume MEMS packaging houses.

\section{Wafer-Level Encapsulation}

Wafer level encapsulation mitigates many of the challenges associated with MEMS packaging, such as dicing, handling, and encapsulation. In this technique, a capping wafer is bonded to the top of a device wafer and when diced, each MEMS chip effectively has a protective lid attached to it. There are several methods that can be used to bond the capping wafer to the device wafer. The wafer bonding can be done using direct bonding, however, the required temperature is about $1000^{\circ} \mathrm{C}^{20}$ and is incompatible with integrated CMOS (complementary metal-oxide semiconductor) control circuitry. Glass-frit or anodic bonding is more commonly used because the processing temperature is between 450 and $500^{\circ} \mathrm{C}^{10}$. However, the glass frit may cause stress in the die if the glass used has a CTE sufficiently different from that of silicon. Anodic bonding requires high voltage, which can also be a disadvantage for integrated systems combining MEMS and IC devices on a single chip ${ }^{3}$. These wafers can also be bonded in a vacuum to produce a vacuum environment inside each device chip, which may be desirable for some applications. Although wafer-level encapsulation has many advantages, it may also add significant cost to device fabrication. However, a cost analysis of fabrication, packaging, and testing may result in a reduction of the overall manufacturing costs due to greatly reduced packaging and testing costs.

\section{Stress}

When polysilicon is deposited, a great deal of stress is created in the film. Most of this stress can be annealed out at a temperature of around $1000^{\circ} \mathrm{C}$, and is most effective if the polysilicon is deposited amorphously and then annealed to form a polycrystalline structure ${ }^{21}$. This creates the lowest stress arrangement with the fewest defects in the polysilicon. The second source of stress results from the die attach material at the interface between the MEMS die and the package substrate. Depending on the die attach material and CTE mismatch between the package and the chip, interfacial stress can develop within a MEMS package ${ }^{22}$. Die stress can be reduced by simply using a low stress die attach material. However, many low stress attaches are also high in outgassing, which can cause other detrimental effects on the MEMS system. Also, low stress die attach materials may creep, leading to stress relaxation.

A major drop in reliability may be caused by excess stress in the package. This stress can be caused by stress inducing fabrication processes, CTE mismatch in the die attach, lid sealing, or shrinkage during the attach curing. The results of stress are that the devices may deform, gear teeth may become misaligned, tensile stress may cause the resonant frequency to increase and result in device breakage, and excessive compressive stress causes long beam elements to buckle. Packaging stresses can induce both offset and scale factor shifts in sensors. In addition, the use of hard solders like AuSn or AuSi can put excessive stress on the delicate components and cause the features, as well as the die itself, to warp or fail ${ }^{23.24}$.

Stress effects also worsen as chip size increases. MEMS chip sizes may be larger than many IC chips since the feature sizes are larger and the devices typically require more die area. This stress can be reduced by using lower modulus die attach materials that deform as the chip and package expand and contract ${ }^{23}$. These low modulus die attach materials may also allow stress relaxation which could be a poor choice because a change in the stress state can lead to changes in device performance. These variations in stress lead to changes in device characteristics such as resonant frequency, offset voltage, and scale factor. Although high stress may be undesirable, it is also undesirable for the stress-state to change over time ${ }^{25}$.

\section{Outgassing}

For die attachment either polymer or metal solders can be used. When polymer, such as epoxies or cyanate esters are used for die attach, water and organic vapors are generated as they cure ${ }^{26}$. The emission of these water and/or organic vapor is called outgassing. Outgassing also occurs after die attachment when die is in the package. 
The water and organic vapors generated in outgassing may then redeposit on the features, in crevices, and on bond pads. This leads to device stiction and corrosion. Stiction prevents the operation of the moving parts, while corrosion can affect the electrical conduction paths by increasing impedance and promoting electrical failures. Die attach materials with a low Young's Modulus, like epoxies, also allow the chip to move during ultrasonic wirebonding, resulting in low bond strength, which has been documented in certain pressure sensors" ${ }^{11}$. These poor wirebonds create reliability issues, as well as increased impedance due to the poor attachment. Possible solutions to outgassing challenges include very low outgassing die attach materials with sufficiently high Young's Modulus and/or the removal of outgassing vapors during die attach curing. For solving the problem of outgassing after die attach when die is in the package, special mechanism/technique could be adapted so that there is chemisorption or physisorption of outgassed materials. One such technique that can be used to remove water and/or organic vapor is use of gettering materials. In our laboratory at the University of Arkansas, we are researching the use of gettering materials in MEMS packaging to solve the problem of corrosion and stiction in MEMS devices.

\section{Testing}

Testing MEMS devices is also an issue. No one wants to package a bad chip; it is too expensive and too time consuming. MEMS devices are difficult to test for several reasons. One reason is that, in the unreleased state, the devices are not free to move, so operational testing of moving features is impossible. In this state, only electrical measurements can be made, such as sheet resistance and conductivity. After releasing, devices can be tested for proper mechanical operation, however, this is after significant time and processing investments in the part. Also, individual dice are time consuming to handle, and are not well suited for automatic test probers as are wafer-scale parts. At the current time, the most efficient testing is done after packaging when the full operation of the parts can be tested, and the parts are easy to handle in packaged form. Any electrical testing that can be done prior to releasing the devices should also be used to eliminate die that have electrical faults from the process stream.

\section{State-of-the-Art in MEMS Packaging}

The present state-of-the-art in MEMS is combining MEMS with ICs and utilizing advanced packaging techniques to create complex MEMS systems. Two different approaches are to use Multi-chip Modules (MCM) and monolithic integration. Monolithic integration is described as the idea of placing CMOS and MEMS on the same chip. The challenge is that the processing steps for CMOS and MEMS are not compatible. For instance, high temperature annealing used in MEMS processing destroys the diffusion profiles and aluminum interconnects used in the CMOS devices. There are three main methods that have been used to create the monolithic integration of CMOS and MEMS: (1) Electronics First (University of California, Berkeley), (2) MEMS in the Middle (Analog Systems), and (3) MEMS First (Sandia National Laboratories) ${ }^{3}$.

One of the most recent efforts in monolithic integration has been Sandia's MEMS First effort in which the MEMS are first fabricated in an etched trench then covered with a sacrificial oxide. After the trench is filled completely with $\mathrm{SiO}_{2}$, the surface is planarized. This flat surface serves as the starting material for the CMOS foundry. The sacrificial oxide covering the MEMS is removed after the CMOS devices are fabricated. This protects the MEMS devices from the CMOS processing steps ${ }^{3.27}$. Figure 5 is a schematic of Sandia's MEMS First approach to monolithic integration. An alternative approach to monolithic integration is the use of $\mathrm{MCM}^{28}$. An IC and a MEMS dice can be placed in the same package, as opposed to the same die, eliminating these processing incompatibilities. However, parasitic effects are ultimately reduced placing the control electronics as close as possible to the MEMS devices.

Advanced packaging techniques like MCM and flip chip are being actively pursued for use with $\mathrm{MEMS}^{29}$. The idea of MCM is that several different MEMS sensors and actuators, or a combination, can be combined into a single package forming complex systems that can perform several functions in a single package. These MCM systems should be modular and any number of them could be constructed from the available MEMS sensors and actuators $^{28}$. This opens the already nearly limitless range of applications for systems of much greater complexity.

A downside to MCM use is the apparent added packaging expense. This signal loss is especially noticeable with some capacitive devices. For some of these devices, the capacitance changes being sensed are less than a femtofarad. A single 100 micron by 100 micron bond pad can add a picofarad of capacitance, thereby swamping the desired signal change ${ }^{30}$. In these applications, one may put detection circuitry right next to the MEMS device in 
order to decrease the effects of parasitic capacitance. The apparently greater cost of packaging is due to the issue of known good die (KGD), or pretested dice, that are known to work before being placed into the MCM. MCMs also have larger packages sizes that do cost more than a single chip package, but fewer packages are needed. Also depending on the MCM requirements, the substrate can be a major part of the packaging costs if it requires multiple layers and high density signal lines. Stress can also be increased from the larger package dimensions ${ }^{13}$. The disposability of MCM packaged systems becomes much less palatable, and the cost of rework more acceptable.

The next state-of-the-art process is the idea of lab-on-chip. This is the concept of several sensors and actuators on a single chip, or in the same package using MCM technology, forming a mixed signal system that will fulfill a function or group of functions. The objective is to realize the size, weight, and cost reductions of microsystems that create significant benefits over many larger systems. Mixed signal in this sense refers to input and/or output signals that can be mechanical, electrical, magnetic, optical, biological, acoustical, chemical, etc. There are several directions researchers are going with the concept of lab-on-chip. There are applications involving chem-lab-on-chip where the object is to create several sophisticated chemical sensors on a single chip ${ }^{30}$. Another application is an optical bench on a chip which utilizes semiconductor lasers, beam splitters, movable mirrors, lenses, etc., to make a miniaturized version of an optical bench ${ }^{31}$. Also, efforts have been directed toward a DNA lab on a chip where the polymerase chain reaction (PCR) is carried out to amplify DNA and then various separation techniques are used to analyze the DNA ${ }^{32}$.

Due to the size of MEMS devices, it is best to have non-contact signal interfaces with the environment. Microdevices can be destroyed by even very small contact forces; however, they can generally survive amazingly high non-contact force levels, such as inertial forces ${ }^{33}$. Mechanical signal interfaces, in the microNewton range, between MEMS devices, such as geartrains, however, are commonplace. The lab-on-chip concept capitalizes on the many possibilities and advantages of MEMS. It is conceivable that an entire control panel of sensors could be reduced to hand-held size. This would have tremendous value in spacecraft and fighter jets where space and weight considerations are critical ${ }^{29}$. On a space expedition like the Pathfinder mission, many more experiments and observations could be made because of the increased number of sensors and instruments that would fit on board.

\section{Summary .}

The role of packaging is to provide a compact housing, as weil as an interface between a device and the outside world. It should protect the chip, while letting it perform its intended function cleanly with very little attenuation or distortion of the signals, such as electrical and/or mechanical and/or optical, etc., in the given environment, and do so at a low cost. The packaging and assembly, as well as the materials used, are integral parts of a microsystem. The total cost of processing and assembly must be taken into account when designing and fabricating a device. Currently, packaging is a significant portion of the total cost of a MEMS device. MEMS packages will be specialty items that will generate higher profit margins, creating new opportunities for small. lowvolume packaging companies. Traditional front-end and back-end packaging become blurred and united in the development of the system. MEMS packaging breaks the paradigms of traditional packaging, and is an exciting field ready for additional wide-spread research and development of new applications. Currently, there is scattered research being performed related to MEMS packaging, for example, in the areas of die attach and outgassing. However, focused efforts on packaging issues such as stiction, stress, and outgassing are essential for reliable implementation of the technology.

\section{Future Directions}

In the future, the field of packaging and integration will be required to consider, not only the packaging of individual devices, but also the seamless integration of electrical (ICs) and/or mechanical (MEMS) and/or optical (integrated optics) and/or chemical, etc. devices. Generic packaging schemes should be developed so that multiple users can select a common package design. MEMS device designers should not be required to also develop custom packages where more generic packages would serve their purposes well. The advanced packaging and integration concepts need to address the packaging of individual devices, as well as multi-signal devices, for synergistic response in the desired environment. In particular, for MEMS devices, based on the current knowledge base, we believe that the packaging and integration could be realized in three ways: hybrid thick film, multichip module (MCM), and monolithic integration. Hybrid thick film is the integration of passive components, such as resistors, 
that can be fabricated using thick-film technologies. MCM is another integration regime that takes existing chips from different signal domains and integrates them into a single package. This increases silicon efficiency, decreases the effects of electrical parasitics, and creates systems that are more compact. Monolithic integration is the most compact form of system integration, but creates the most processing challenges. With CMOS and MEMS processing incompatibilities already mentioned, it takes creative processing solutions to create functional systems. The future of MEMS is most assuredly the marriage of mechanical devices to control electronics and optics. There will be interaction between micromechanical, optical, chemical, biological, and electrical systems to perform tests and analysis in many domains never before possible. Examples of work already in progress is gene therapy, DNA replication, sensors waveguides. This necessitates even more interaction between disciplines, and alignment of disciplines never before paired together. It is an exciting field, however, packaging is the link that brings novel laboratory devices into the commercial, military, and medical fields.

\section{Acknowledgments}

The authors of this paper would like to acknowledge Defense Advanced Research Project Agency (DARPA) as well as National Science Foundation (NSF) for their financial support of MEMS packaging research and Sandia National Laboratories' Micromachine Initiative for technical direction and instrumentation. Sandia is a multiprogram laboratory operated by Sandia Corporation, a Lockheed Martin Company, for the United States Department of Energy under contract DE-AC-04-94AL85000. We would also like to acknowledge Jim Smith and Bill Conley from Sandia for their useful discussions.

\section{References}

1. J. J. Sniegowski, "Multi-level Polysilicon Surface-micromachiningTechnology: Applications and Issues,"(Invited Paper) ASME 1996 International Mechanical Engineering Congress and Exposition, Proc. of the ASME Aerospace Division, November 1996, Atlanta, GA, AD-Vol. 52, pp. 751-759.

2. J. J. Sniegowski, "Moving the World with Surface Micromachining," Solid State Technology, February 1996, pp. 83-90.

3. Sandia National Laboratories Introductory MEMS Short Course, June 29 - July 1, 1998.

4. J. J. Sniegowski and E. J. Garcia (1995). "Microfabricated Actuators and their Application to Optics", Proceedings of Photonics West ' 95.

5. D. L. Hetherington and J. J. Sniegowski, "Improved Polysilicon Surface-micromachined Micromirror Devices using Chemical-mechanical Polishing", International Symposium on Optical Science, Engineering, and Instrumentation, SPIE's 43rd Annual Meeting, San Diego, CA, July 1998.

6. B. Kloeck and N.F. de Rooij, "Mechanical Sensors", Semiconductor Sensors, ed. S. M. Sze, J Wiley, 1994, pp. 153-199.

7. Peeters, "Challenges in Commercializing MEMS", IEEE Computational Science and Engineering, 1997, pp. 4448.

8. Sandia National Laboratories, Albuquerque, NM, Micromachine Initiative Webpage, http://www.mdl.sandia.gov/Micromachine/summit5.html.

9. MCNC, Research Triangle Park, NC, Cell Library Webpage, http://mems.mcnc.org/camel.html.

10. D.J. Monk, T. Maudie, D. Stanerson, J. Wertz, G. Bitko, J. Matkin, and S. Petrovic, "Media Compatible Packaging and Environmental Testing of Barrier Coating Encapsulated Silicon Pressure Sensors", Solid State Sensor and Actuator Workshop, Hilton Head, South Carolina, June 2-6, 1996, pp.36-41.

11. D.J. Monk and M.K. Shah, "Packaging and Testing Considerations of Bulk Micromachined, Piezoresistive Pressure Sensors", Motorola Sensor Products Division.

12. H. de Lambilly, R. Grace, and K. Sidhu, "Package or Perish", Proceedings of the 1996 Sensors Expocon, 1996, p.275.

13. W.D. Brown, ed., Advanced Electronic Packaging: With Emphasis on Multichip Modules, IEEE Press, New York, 1999, pp. 3-8, 16-22, 35-41.

14. R. Legtenberg, A. C. Tilmans, J. Elders, and M. Elwenspoek, "Stiction of surface micromachined structures after rinsing and drying: model and investigation of adhesion mechanisms," Sensors and Actuators, A: Physical, 
43(1-3), pp. 230-238, 1994.

15. G. T. Mulhern, D. S. Soane, and R. T. Howe, "Supercritical Carbon Dioxide Drying of Microstructures", the $7^{\text {th }}$ International Conference of Solid-State Sensors and Actuators, Transducers '93, pp. 296-299, 1993.

16. M. R. Houston, R. Maboudian, and R.T. Howe, "Ammonium flouride anti-stiction treatments for polysilicon microstructures", Digest of Technical Papers, 1995 International Conference on Solid-State Sensors and Actuators, Transducers '95, paper 45, 1995.

17. W. C. Tang, T-C.H. Nguyen, and R.T. Howe, "Laterally driven polysilicon resonant microstructures", 1989 International Workshop on Micro Electromechanical Systems (MEMS '89), p. 53, 1989.

18. M.H. Kiang, O. Solgaard, K.Y. Lau, and R.S. Muller, "Electrostatic Combdrive-Actuated Micromirrors for Laser-Beam Scanning and Positioning", Journal of Microelectromechanical Systems, vol. 7, no. 1, March 1998, pp. 27-37.

19. Dynatex Inc. Santa Rosa, CA, Webpage, http://www.dynatex.com.

20. J. B. Lasky, S. R. Stiffler, F. R. White, and J. R. Abernathy, "Silicon-on-insulator (SOI) by Bonding and Etch-Back", Technical Digest, 1985 IEEE International Electron Device Meeting pp.684-687.

21. H. Guckel, D. W. Burns, C. R. Rutigliano, D. K. Showers, and J. Uglow, "Fine-grained polysilicon and its application to planar pressure transducers", Technical Digest, The 4th International Conference on Solid-State Sensors and Actuators, 1987 pp. 277-282.

22. M.L. Kniffin and M. Shah, "Packaging for Silicon Micromachined Accelerometers", The International Journal of Microcircuits and Electronic Packaging, first quarter 1996, vol. 19 no. 1, pp.75-86.

23. B. I. Chandran, "Determination and Utilization of AuSn Creep Properties for Bonding Devices with Large CTE Mismatches", University of Arkansas, 1996.

24. M. D. Brown, "Investigation of flip-chip die attachment to diamond heat spreaders", University of Arkansas, 1998.

25. A. C. McNeil, "A Parametric Method for Linking MEMS Package and Device Models", 1998 Solid State Sensors and Actuators Workshop, Hilton Head '98, June 1998, pp.166-169.

26. I.Y. Chien and M.N. Nguyen, "Low stress polymer die attach adhesive for plastic packages", Electronic Engineering, February 1995, pp. 41-46.

27. J. H. Smith, S. Montague, J. J. Sniegowski, J. R. Murray, and P. J. McWhorter, "Embedded Micromechanical Devices for the Monolithic Integration of MEMS with CMOS", Technical Digest, The 1995 International Electron Device Meeting pp. 609-612.

28. J.T. Butler, V.M. Bright, and J.T. Comtois, "Advanced Multichip Module Packaging of Microelectromechanical Systems", Transducers '97, 1997 International Conference on Solid-State Sensors and Actuators, Chicago, June 16-19, 1997, pp. 261-264.

29. J. Lyke, "Packaging Technologies for Space-Based Microsystems and their Elements", Packaging Technologies, pp.133-180.

30. S.T. Picraux and P. J. McWhorter, "The Broad Sweep of Integrated Microsystems", IEEE Spectrum, vol. 35 no. 12, December 1998, pp.24-33.

31. J. H. Smith, M. S. Rodgers, J. J. Sniegowski, S. L. Miller, D. L. Hetherington, P. J. McWhorter, and M. E. Warren, "Micro-electro-optical Devices in a Five-level Polysilicon Surface-micromachining Technology", Proceedings of Micromachined Devices and Components IV, Proceedings SPIE, vol. 3514, September 1998, pp.4249.

32. A. T. Wooley, D. Hadley, P. Landre, A. J. de Mollo, R. A. Mathies, and M. A. Northrup, "Functional Integration of PCR Amplification and Capillary Electrophoresis in a Microfabricated DNA device", Anal. Chem, vol. 70, 1998, p. 158.

33. T. G. Brown and B. S. Davis, "Dynamic High-G Loading of MEMS Sensors: Ground and Flight Testing", Materials and Device Characterization in Micromachining, Proc. of SPIE, vol. 3512, September 1998, pp. 228-235.

\section{About the Authors}

Ajay P. Malshe, Ph.D. (1992), is an Assistant Professor at the Department of Mechanical Engineering and an adjunct faculty at the High Density Electronics Center (HiDEC), Department of Electrical Engineering, University of Arkansas, Fayetteville, AR. His background is materials science and engineering, and has authored more than 60 referred publications and holds three patents. His two distinct fields of research interest are electronic-packaging, and 
materials and manufacturing. The electronic packaging related subjects of his interest are nano and micro systems packaging and integration, high-density MCM electronics packaging, 3D packaging and thermal management. He is active in IMAPS, ASME, ASEE and other related professional societies.

Chad B. O'Neal received his B.S. from the Department of Mechanical Engineering at the University of Arkansas in 1998 and is currently pursuing his M.S. in mechanical engineering at the High Density Electronic Center (HiDEC) located at the University of Arkansas, Fayetteville. Mr. O'Neal is currently studying the area of MEMS packaging, specifically the effects of die attachment stress induced onto the devices and the functionality of the MEMS devices after packaging. His research of MEMS packaging is in collaboration with Sandia National Laboratories. He currently has two publications.

Sushila Singh received Ph.D. from Department of Electronic Science, University of Poona, Pune, India, in 1996. After Ph.D. she worked as a Project Associate for Optoelectronics, a Division of the Society for Applied Microwave Electronics Engineering Research (SAMEER), Department of Electronics, Government of India. Presently she is a visiting research assistant professor at the Department of Physics, University of Arkansas, Fayetteville, AR. Her three distinct fields of research interest are electronic packaging of MEMS, optical interconnects for electronic packaging and optoelectronic devices for communication and computation. She has authored over 20 publications.

William D. Brown received the B.S. degree in Electrical Engineering from the University of Arkansas, Fayetteville, in 1969, the M.S. degree from Pennsylvania State University, University Park, in 1970; and the Ph.D. degree from the University of New Mexico, Albuquerque, in 1975. From 1969 to 1977, he was a Member of the Technical Staff at Sandia National Laboratories in Albuquerque, NM. In 1977, he joined the faculty of the Electrical Engineering Department at the University of Arkansas where he presently holds the rank of University Professor and serves as the Associate Dean for Research in the College of Engineering. His research interests include microelectronic materials and devices, and solid-state and semiconductor physics. Dr. Brown's research has resulted in 96 technical journal publications, 186 conference/workshop presentations, 83 technical conference proceedings articles, and 16 educational conference proceedings articles. He has contributed to and edited the books, Electronic Manufacturing Processes published by Prentice Hall, and Nonvolatile Semiconductor Memory Technology and Advanced Electronic Packaging: With Emphasis On Multichip Modules, both published by the IEEE PRESS. He presently serves on the Executive Committee of the Board of Directors of the Electrochemical Society. He is a registered Professional Engineer in the State of Arkansas and holds memberships in IEEE, IMAPS, ECS, MRS, AVS, the Arkansas Academy of Science, and the American Society of Engineering Educators (ASEE).

William P Eaton received the M.S. degree in Materials Science from the University of California at Davis in 1993, and the Ph.D. degree in Electrical Engineering from the University of New Mexico, Albuquerque, in 1997. He is currently in the Reliability Physics department at Sandia National Laboratories, Albuquerque, NM, working on MEMS reliability. He has been actively doing MEMS research since 1990 , working on projects in wafer bonding, bulk micromachining, surface-micromachined pressure sensors, release etch modeling, reliability, device characterization, and packaging.

William M. Miller manages the Reliability Physics Department at Sandia National Laboratories. He received his B.S. (1979) in physics from the University of Washington and his M.S. (1980) and Ph.D. (1984) in physics from the University of Illinois. He has been employed by Sandia since 1984, working on silicon nitride non-volatile memories, silicon-on-insulator (SOI) technology and metallization processes. Since 1989 he has worked on IC reliability issues, with an emphasis on dielectric breakdown and MicroElectroMechanical Systems (MEMS). He became the manager of Sandia's Reliability Physics Department in 1992. During his career he has published 17 technical papers and has mad scores of presentations on a wide range of topics. He currently holds one US patent and has another pending in the area of MEMS. 
Table 1. Current packaging parameters, challenges, and suggested possible solutions for MEMS.

\begin{tabular}{|c|c|c|}
\hline $\begin{array}{l}\text { Packaging } \\
\text { Parameters }\end{array}$ & Challenges & Possible Solutions \\
\hline $\begin{array}{l}\text { Release etch and } \\
\text { dry }\end{array}$ & $\begin{array}{l}\text { Prevention of device stiction, } \\
\text { washing away of parts during } \\
\text { release, must release parts } \\
\text { individually after dicing }\end{array}$ & $\begin{array}{l}\text { Freeze drying, supercritical } \mathrm{CO}_{2} \text { drying, roughening of } \\
\text { contact surfaces, coatings or processes that reduce } \\
\text { surface tension, use of dimples, develop a dicing } \\
\text { process in which parts can be released before dicing, } \\
\text { possibly wafer cleaving or laser sawing }\end{array}$ \\
\hline Dicing/Cleaving & $\begin{array}{l}\text { Eliminating contamination } \\
\text { caused by cooling fluid and } \\
\text { particulates during wafer sawing }\end{array}$ & $\begin{array}{l}\text { Release dice after dicing, cleaving of wafers, laser } \\
\text { sawing, wafer level encapsulation }\end{array}$ \\
\hline Die handling & $\begin{array}{l}\text { Damages top die face contact } \\
\text { region }\end{array}$ & $\begin{array}{l}\text { Fixtures that hold MEMS dice by sides rather than top } \\
\text { face, such as collets that fit existing pick-and-place } \\
\text { equipment }\end{array}$ \\
\hline Stress & $\begin{array}{l}\text { Abating performance degradation } \\
\text { and resonant frequency shifts, } \\
\text { curling of thin film layers, } \\
\text { misalignment of device features }\end{array}$ & $\begin{array}{l}\text { Low modulus, low creep die attach material, annealing, } \\
\text { die attach materials with CTE similar to that of silicon } \\
\text {. }\end{array}$ \\
\hline Outgassing & $\begin{array}{l}\text { Stiction, corrosion, outgassing of } \\
\text { organic solvents from polymeric } \\
\text { die attach materials }\end{array}$ & $\begin{array}{l}\text { Low outgassing epoxies, cyanate esters, low modulus } \\
\text { solders, new die attach materials, removal of outgassing } \\
\text { vapors }\end{array}$ \\
\hline Testing & $\begin{array}{l}\text { Applying non-electric stimuli to } \\
\text { devices, testing moving device } \\
\text { features before release, unable to } \\
\text { release parts before dicing }\end{array}$ & $\begin{array}{l}\text { Electrical test structures to mimic non-electrical } \\
\text { functions, modify (where possible) wafer-scale probers } \\
\text { to do non-electrical tests, cost effective, high } \\
\text { throughput, and parallel packaged device test systems }\end{array}$ \\
\hline
\end{tabular}


Figure 1. A Sandia National Laboratories micromachined geartrain. The gear is approximately 80 microns in diameter.

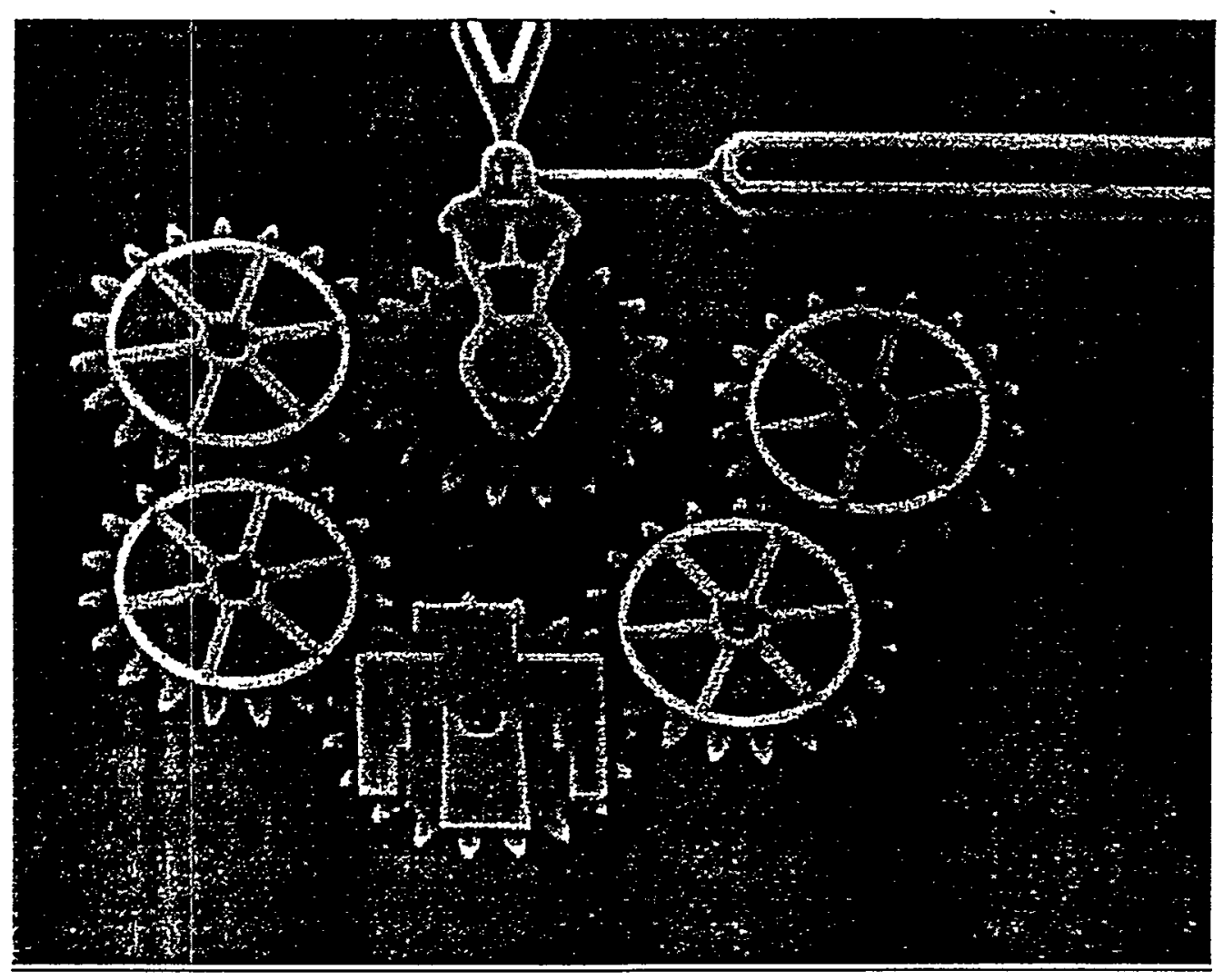


Figure 2. Schematic of an (a) IC package [13] (b) MEMS pressure sensor package [11]. The IC is encapsulated, where the MEMS is open to the air.
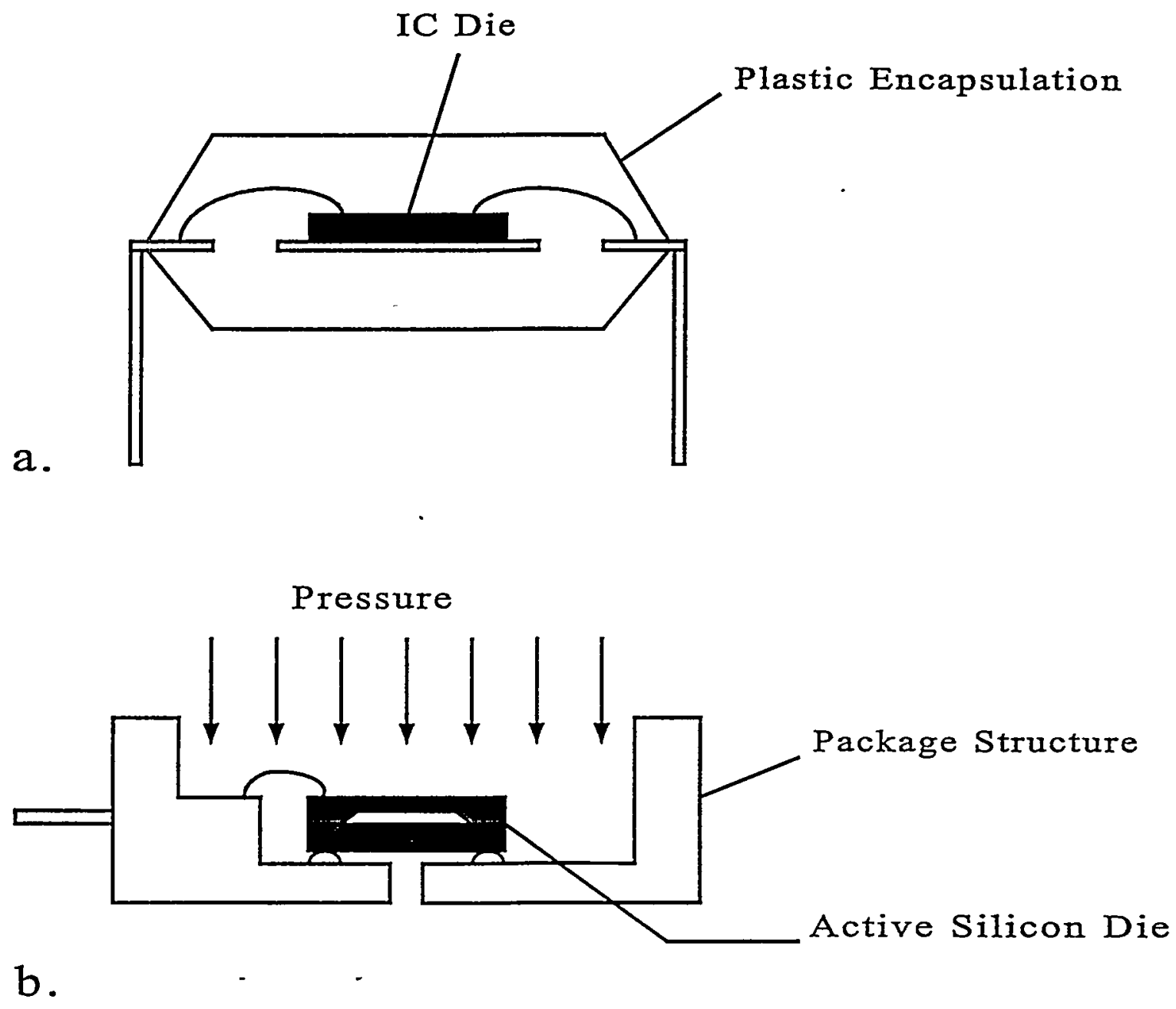
Figure 3. Beam-type element displaying the effect of stiction.

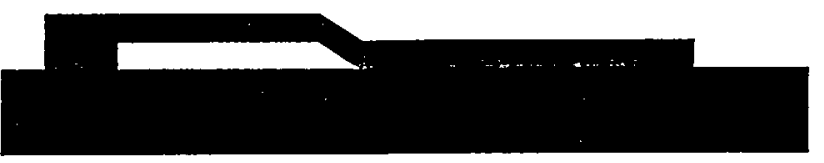


Figure 4. An example of dimples used to prevent stiction.

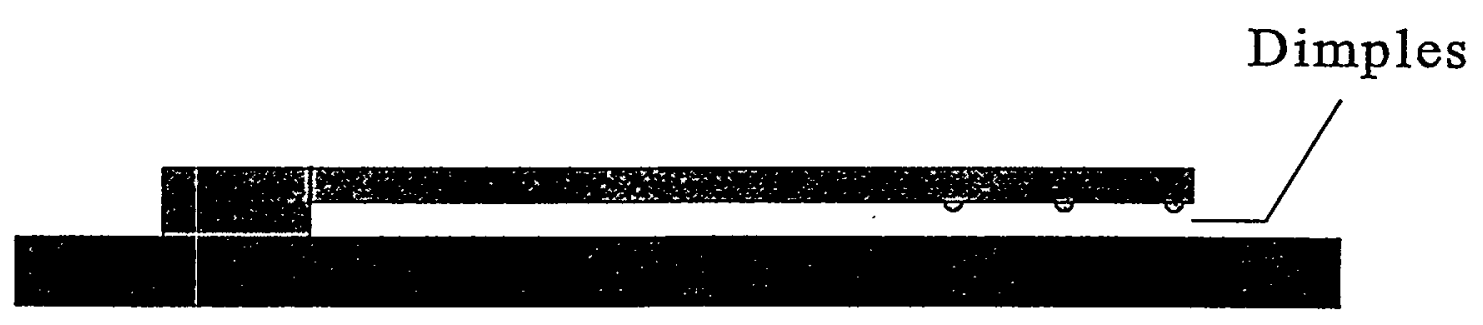


Figure 5. Cross-sectional schematic of Sandia's MEMS First approach to monolithic integration [3], [27].

\section{CMOS}

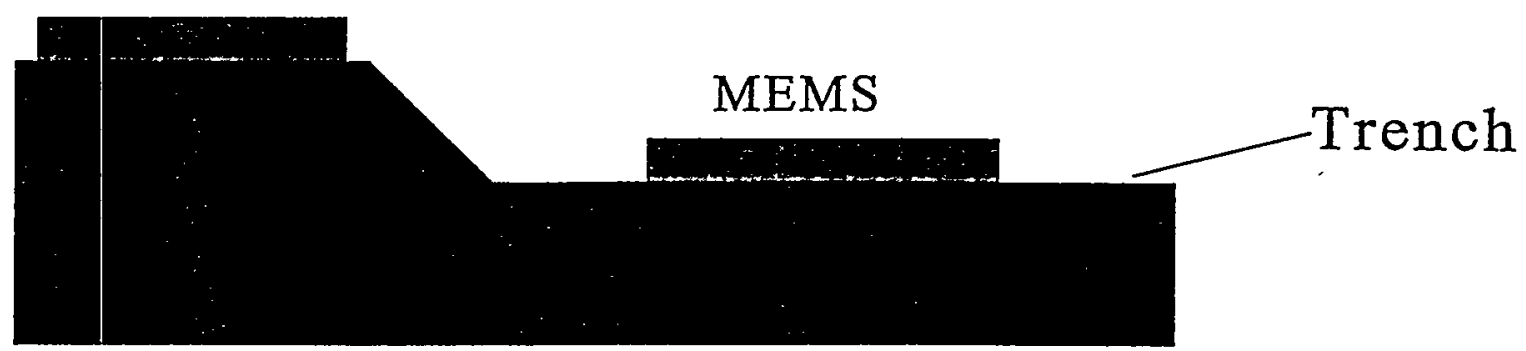


Figure 5. Cross-sectional schematic of Sandia's MEMS First approach to monolithic integration [3], [27].

\section{CMOS}

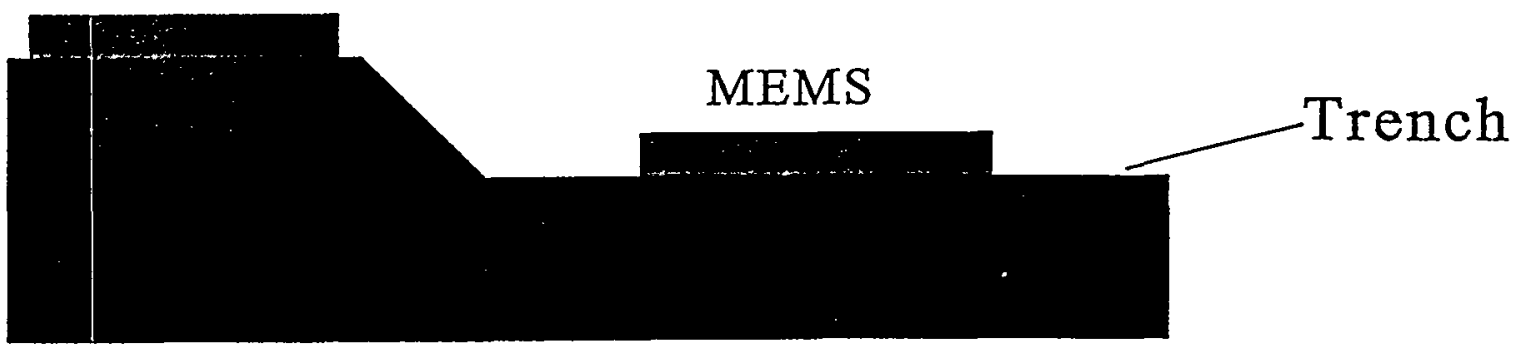

